\title{
Early data on the performance of a combined SARS-CoV-2 spike-nucleocapsid antibody lateral flow immunoassay device compared with a nucleocapsid-only device
}

\author{
Authors: Christian Linares, ${ }^{A}$ Felicity Ryan, ${ }^{B}$ Hannah Lowe ${ }^{B}$ and Samuel Moses ${ }^{B}$
}

\section{Introduction}

There is a critical need for reliable antibody detection methods in order to study and evaluate the public health and clinical response to the ongoing coronavirus disease 2019 (COVID-19) pandemic. ${ }^{1}$ Lateral flow immunoassay (LFIA) devices potentially offer the prospect of rapid point-of-care testing (POCT), but the performance of these devices must be evaluated for robustness before they can be adopted for routine clinical and public health use..$^{2-4}$ Furthermore, as of when this study was conducted in May 2020, there were no published studies of these LFIA devices for severe acute respiratory syndrome coronavirus 2 (SARS-CoV-2) antibodies.

\section{Materials and methods}

In the present study, anonymised, residual stored plasma and serum specimens from SARS-CoV-2 RNA-positive $(\mathrm{n}=131)$ and presumed (pre-COVID-19) or RNA-negative $(n=37)$ patients were taken from various time points with respect to the onset of symptoms. All 168 anonymised specimens were tested for IgM and IgG using the Hangzhou AllTest 2019-nCoV IgG/IgM rapid test cassette and the Abbexa COVID-19 IgG/ IgM rapid test kit.

\section{Results and discussion}

There were great disparities in the responses to IgM and IgG between the two devices, but sensitivity for IgG detection improved over time from symptom onset (Fig 1). IgM sensitivity ranged from $12.90 \%$ (95\% confidence interval (CI) $3.63-29.83$ ) to $68 \%$ (95\% CI 46.50-85.05), depending on the date of symptom onset and the device. Regarding IgG, the Abbexa device outperformed the Hangzhou device at all cumulative timeline brackets, with sensitivity of $97.87 \%$ (95\% CI 88.71-99.95) for Abbexa versus 68.09\% (95\% CI 52.88-80.91) for Hangzhou for samples beyond 21 days from symptom onset (Table 1). Day 21 was therefore chosen as

Authors: 'Auy's and St Thomas' NHS Foundation Trust, London, UK; 'Bast Kent Hospitals University NHS Foundation Trust, UK
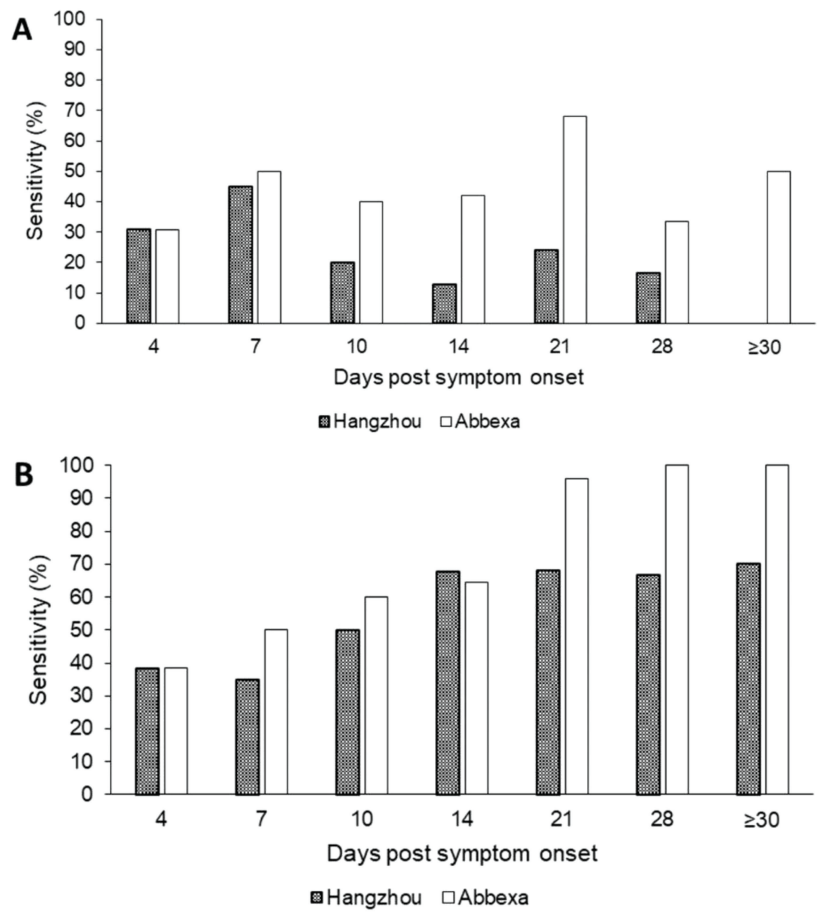

Fig 1. Development of immunoglobulins over the convalescent timeline using Hangzhou and Abbexa LFIA devices. a) IgM. b) IgG.

the cut-off for ascertaining test performance characteristics, beyond which the specificity was 100\% (95\% CI 90.51100.00) for both Abbexa and 97.30 (95\% CI 85.84-99.93) for Hangzhou. Negative predictive value was 0.97 (95\% CI 0.84-1.00) for Abbexa versus 0.71 (95\% CI 0.61-0.79) for Hangzhou.

\section{Conclusion}

Based on this limited dataset, the performance characteristics of the Abbexa LFIA device were substantially better than those of the Hangzhou device. Applying a 21-day cut-off for the Abbexa device revealed performance that was very close 
Table 1. Comparative performance characteristics of Hangzhou and Abbexa LFIA devices for detecting SARSCoV-2 IgM and IgG in PCR-positive patients at varying times following symptom onset (with $95 \%$ confidence intervals)

\begin{tabular}{lcccc}
\hline From $D_{0}$ of symptom onset (168) & & & & \\
Sensitivity (\%) & $22.14(15.35-30.22)$ & $46.56(37.81-55.48)$ & $57.25(48.32-65.85)$ & $70.99(62.42-78.59)$ \\
Specificity (\%) & $97.30(85.84-99.93)$ & $97.30(85.84-99.93)$ & $97.30(85.84-99.93)$ & $100.00(90.51-100.00)$ \\
PPV & $0.97(0.80-1.00)$ & $0.98(0.90-1.00)$ & $0.99(0.92-1.00)$ & 1.00 \\
NPV & $0.26(0.24-0.28)$ & $0.34(0.30-0.38)$ & $0.39(0.34-0.44)$ & $0.49(0.43-0.56)$ \\
\hline Z7 days from symptom onset (155) & & & & \\
Sensitivity (\%) & $21.19(14.20-29.67)$ & $48.31(39.01-57.69)$ & $59.32(49.89-68.27)$ & $74.58(65.74-82.14)$ \\
Specificity (\%) & $97.30(85.84-99.93)$ & $97.30(85.84-99.93)$ & $97.30(85.84-99.93)$ & $100.00(90.51-100.00)$ \\
PPV & $0.96(0.78-0.99)$ & $0.98(0.89-1.00)$ & $0.99(0.91-1.00)$ & 1.00 \\
NPV & $0.28(0.26-0.30)$ & $0.37(0.33-0.41)$ & $0.43(0.37-0.48)$ & $0.55(0.48-0.63)$ \\
\hline Z14 days from symptom onset (115) & & & & \\
Sensitivity (\%) & $15.38(8.21-25.33)$ & $50.00(38.46-61.54)$ & $67.95(56.42-78.07)$ & $84.62(74.67-91.79)$ \\
Specificity (\%) & $97.30(85.84-99.93)$ & $97.30(85.84-99.93)$ & $97.30(85.84-99.93)$ & $100.00(90.51-100.00)$ \\
PPV & $0.92(0.62-0.99)$ & $0.98(0.85-1.00)$ & $0.98(0.88-1.00)$ & 1.00 \\
NPV & $0.35(0.33-0.38)$ & $0.48(0.42-0.54)$ & $0.59(0.51-0.67)$ & $0.76(0.65-0.84)$ \\
\hline Z21 days from symptom onset (84) & & & & \\
Sensitivity (\%) & $17.02(7.65-30.81)$ & $55.32(40.12-69.83)$ & $68.09(52.88-80.91)$ & $97.87(88.71-99.95)$ \\
Specificity (\%) & $97.30(85.84-99.93)$ & $97.30(85.84-99.93)$ & $97.30(85.84-99.93)$ & $100.00(90.51-100.00)$ \\
PPV & $0.89(0.51-0.98)$ & $0.96(0.79-.99)$ & $0.97(0.82-1.00)$ & 1.00 \\
NPV & $0.48(0.45-0.52)$ & $0.63(0.63-0.83)$ & $0.71(0.61-0.79)$ & $0.97(0.84-1.00)$ \\
\hline 228 days from symptom onset (59) & & & & \\
Sensitivity (\%) & $9.09(1.12-29.16)$ & $40.91(20.71-63.65)$ & $68.18(45.13-86.14)$ & $100.00(84.56-100.00)$ \\
Specificity (\%) & $97.30(85.84-99.93)$ & $97.30(85.84-99.93)$ & $97.30(85.84-99.93)$ & $100.00(90.51-100.00)$ \\
PPV & $0.67(0.16-0.95)$ & $0.90(0.55-0.99)$ & $0.94(0.68-0.99)$ & 1.00 \\
NPV & $0.64(0.61-0.67)$ & $0.73(0.66-0.80)$ & $0.84(0.74-0.90)$ & 1.00 \\
\hline & & & &
\end{tabular}

to meeting the minimum sensitivity and specificity thresholds (98\%) set by the UK Medicines and Healthcare Products Regulatory Agency (MHRA). ${ }^{5}$ The Abbexa device captures antibodies against both SARS-CoV-2 spike and nucleocapsid proteins, as opposed to Hangzhou, which targets only the nucleocapsid protein. ${ }^{2,6-8}$ We therefore propose that spike glycoprotein antibodies be considered as an additional moiety to test as part of the standard diagnostic approach towards SARS-CoV-2 antibody profiling ${ }^{9-10}$ to improve clinical sensitivity and potentially specificity, pending follow-up studies to confirm this approach.

\section{Conflicts of interest}

None declared.

\section{References}

1 Li Z, Yi Y, Luo X et al. Development and clinical application of a rapid IgM-IgG combined antibody test for SARS-CoV-2 infection diagnosis. J Med Virol 2020;1002:1-7.

2 Abbexa. COVID-19 IgG/IgM Rapid Test Kit: Version 8.0.0. Cambridge: Abbexa, UK. www.abbexa.com/pdf/Manual/abx294171. pdf [Accessed 29 June 2020].

3 Vashist S. In vitro diagnostic assays for COVID-19: recent advances and emerging trends. Diagnostics (Basel) 2020;10:202.
4 Department of Health and Social Care. National technical validation process for manufacturers of SARS-CoV-2 (COVID-19) tests. GOV. UK, 2020. www.gov.uk/government/publications/assessment-andprocurement-of-coronavirus-covid-19-tests/coronavirus-covid19-serology-and-viral-detection-testing-uk-procurement-overview [Accessed 26 June 2020].

5 Medicines and Healthcare products Regulatory Agency. Medical Device Alert MDA/2020/015. MHRA, 2020. www.gov.uk/drugdevice-alerts/results-from-laboratory-based-tests-for-covid-19-antibodies-using-capillary-blood-sample-collection-kits-may-not-bereliable-mda-2020-015 [Accessed 08 June 2020].

6 Walls AC, Park YJ, Tortorici MA et al. Structure, function, and antigenicity of the SARS-CoV-2 Spike Glycoprotein. Cell 2020;181:281-92.

7 Indwiani A, Ysrafil. Severe acute respiratory syndrome coronavirus 2 (SARS-CoV-2): an overview of viral structure and host response. Diabetes Metab Syndr 2020;14:407-12.

8 Wu JL, Tseng WP, Lin CH et al. Four point-of-care lateral flow immunoassays for diagnosis of COVID-19 and for assessing dynamics of antibody responses to SARS-CoV-2.J Infect 2020;81:435-42.

9 Egner W. Verification and validation methodology and sample sets for evaluation of assays for SARS-CoV-2 (COVID-19). The Royal College of Pathologists, 2020. www.rcpath.org/uploads/assets/541a45236058-4424-81c119dd2ab0febb/Verification-validation-of-samplesets-assays-SARS-CoV-2.pdf [Accessed 26 June 2020].

10 Nandini S, Jeremiah SS, Ryo A. Interpreting diagnostic tests for SARS-CoV-2. JAMA 2020;323:2249-51. 